\title{
Torque and Twist against Superlubricity
}

\author{
Alexander E. Filippov, ${ }^{1}$ Martin Dienwiebel, ${ }^{2,3}$ Joost W. M. Frenken, ${ }^{2}$ Joseph Klafter, ${ }^{4}$ and Michael Urbakh ${ }^{4}$ \\ ${ }^{1}$ Donetsk Institute for Physics and Engineering of NASU, 83144, Donetsk, Ukraine \\ ${ }^{2}$ Kamerlingh Onnes Laboratory, Leiden University, 2300 RA Leiden, The Netherlands \\ ${ }^{3}$ Fraunhofer Institute for Mechanics of Materials, 79108 Freiburg, Germany \\ ${ }^{4}$ School of Chemistry, Tel Aviv University, 69978 Tel Aviv, Israel
}

(Received 30 August 2007; published 29 January 2008)

\begin{abstract}
Superlubricity between incommensurate surfaces provides a desired low-friction state essential for the function of small-scale machines. Here we demonstrate experimentally and theoretically that superlubricity in contacts lubricated by lamellar solids might be eliminated due to torque-induced reorientation coupled to lateral motion. We find that the possibility of reorientation always leads to stabilization of a high frictional state which corresponds to a commensurate configuration.
\end{abstract}

DOI: 10.1103/PhysRevLett.100.046102

PACS numbers: 68.35.Af, 07.79.Sp, 46.55.+d, 68.37.Ps

The practical importance and the relevance to basic scientific questions have motivated studies towards understanding the conditions which lead to superlow friction. A mechanism for superlow dry friction, which arises from the structural incompatibility of two contacting solids, was first suggested by Hirano and Shinjo [1,2]. This phenomenon is also referred to as superlubricity. However, incommensurability of the interfaces results in a cancellation of only one of the channels of energy dissipation, which originates from a stick-slip instability. Other dissipative processes, such as electronic and phononic friction, etc., still persist, and therefore even in the case of complete incommensurability the net friction force will not be identical to zero. Nonetheless, typical to the superlubricity state is a reduction of the friction force by orders of magnitude.

Detailed experimental studies of superlubricity have been performed recently by Dienwiebel et al. [3-5], who measured friction between a graphite flake attached to the tip of a frictional force microscope (FFM) and an atomically flat graphite surface. Superlow friction forces $(<50 \mathrm{pN})$ have been found for most relative orientations of the flake and the substrate, for which the contacting surfaces find themselves in incommensurate states. For narrow ranges of orientations corresponding to commensurate contacts, stick-slip motion was observed and friction was high (typically $250 \mathrm{pN}$ ). A few earlier experiments $[6,7]$ also provided indications of the superlubricity phenomenon in dry friction.

Measuring friction between graphite flakes and an underlying graphite surface we found that the "lifetime" of a superlubric state can be finite and therefore, superlow friction does not persist. We demonstrate that the dominating contribution to such residual friction in contacts lubricated by lamellar solids may stem from torque-induced reorientation of the flakes attached to the tip. Dynamics of the flake reorientation result in irregular sharp peaks of the lateral force shown in Fig. 1, which are observed during the restoration of the high friction state. The lifetime may depend on the structure of the tip and the density of defects on the graphite surface. Our calculations show that the possibility of flake rotation always stabilizes the high frictional state which corresponds to the commensurate configuration of the contacting surfaces in relative motion. Reorganization of a sliding layer leading to a locking in a "commensurate" state has been previously observed in molecular dynamics simulations [8]. A superlow friction
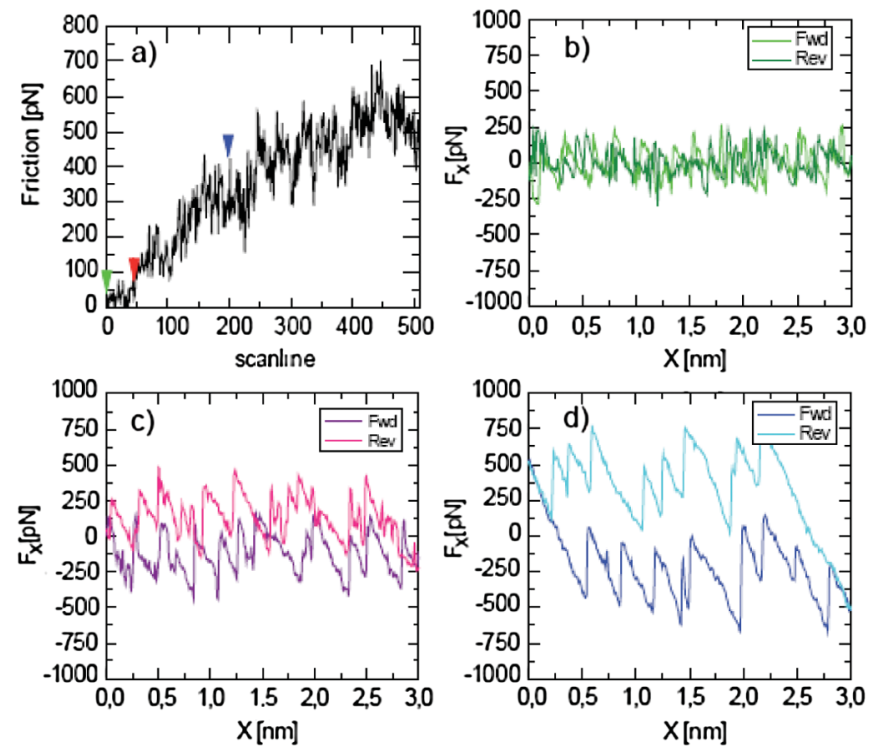

FIG. 1 (color). Measurements of the lateral force between a tungsten tip with a graphite nanoflake attached [3-5] and a highly oriented, pyrolytic graphite substrate. Results were obtained with high-resolution FFM [28] at a scan speed of $30 \mathrm{~nm} / \mathrm{s}$ under a constant positive normal force of $24.3 \mathrm{nN}$. (a) Friction force (average lateral force) as function of time (scan line number); the arrows indicate the locations of the three individual friction loops in (b) -(d); (b) scan line 1 (green arrow); (c) scan line 45 (red arrow); (d) scan line 195 (blue arrow). One scan line equals to $6 \mathrm{~nm}$ ( $3 \mathrm{~nm}$ forward and backward) or $0.2 \mathrm{~s}$. The distance between subsequent scan lines equals $0.024 \mathrm{~nm}$. 
between two incommensurate surfaces may be also eliminated due to the presence of adsorbed molecules between the surfaces [9-12], or the elasticity of the contacting solids [13].

Beyond explaining the results of FFM experiments on graphite, this consideration has important implications for understanding the macroscopic properties of graphite and other solid lamellar lubricants $[14,15]$. Transmission electron microscopy observations [14] on $\mathrm{MoS}_{2}$ indicated that also in a macroscopic sliding contact of lamellar solids rotated flakes are created.

In order to demonstrate the effect of temporal reorientation of the flake on friction, we propose a generalization of the Tomlinson model to include the in-plane rotations of the rigid flake pulled along the surface. The Tomlinson model has proven powerful in describing the dynamics of friction [16,17]. Previous generalizations of the original, one-dimensional Tomlinson model, which included effects of two-dimensional structure of surfaces, lateral-normal coupling, and thermal fluctuations [18-24], marked new steps toward understanding and implementation of frictional phenomena. In particular they led to the introduction of frictional imaging of interfaces [18], mechanical control of friction [25,26], and thermolubricity [24].

Introducing coupling between the lateral motion and inplane rotation of the flake which is attached to the FFM tip and driven along the surface, leads to the following coupled Langevin equations:

$$
m \ddot{\boldsymbol{r}}_{c}+\nabla_{r_{c}} U_{\text {flake }}\left(\boldsymbol{r}_{c}, \phi\right)+\gamma \dot{\boldsymbol{r}}_{c}+K\left(\boldsymbol{r}_{c}-\mathbf{V} t\right)=\mathbf{f}_{r_{c}}(t)
$$

$$
I \ddot{\phi}+\nabla_{\phi} U_{\text {flake }}\left(\boldsymbol{r}_{c}, \phi\right)+\Gamma \dot{\phi}-F_{0} \operatorname{sgn}(\dot{\phi})=f_{\phi}(t) .
$$

Here the first equation describes the translational motion of the center of mass of the rigid flake of an effective mass $m$, whose position is given by the two-dimensional coordinate $\boldsymbol{r}_{c}=\left(x_{c}, y_{c}\right)$, and the second one describes the rotation of the flake having an effective moment of inertia $I$ in the plane $(x, y)$ with respect to the point of its attachment to the tip. We assume that the flake is attached to the tip in its center of mass, and $\phi$ defines the time-dependent rotational (misfit) angle [see Fig. 2(a)]. The tip (and thereby the flake center) is coupled to the FFM support by springs $K_{x}=K_{y}=K$ in the $x$ and $y$ directions, and the support moves with a constant velocity at an angle $\theta$ with respect to the $x$ axis, $\boldsymbol{V}=(V \cos \theta, V \sin \theta)$. In our experiments and simulations we kept $\theta=70^{\circ}$. The parameters $\gamma$ and $\Gamma$ are responsible for the dissipation of the flake's kinetic energy due to the translational and rotational motion, respectively. The graphite flake is treated as a rigid body, and the $\mathrm{N}$-atom flake layer contacting with the substrate is modeled as a finite lattice, composed of hexagonal carbon rings [see Fig. 2(a)]. The interaction between a single carbon
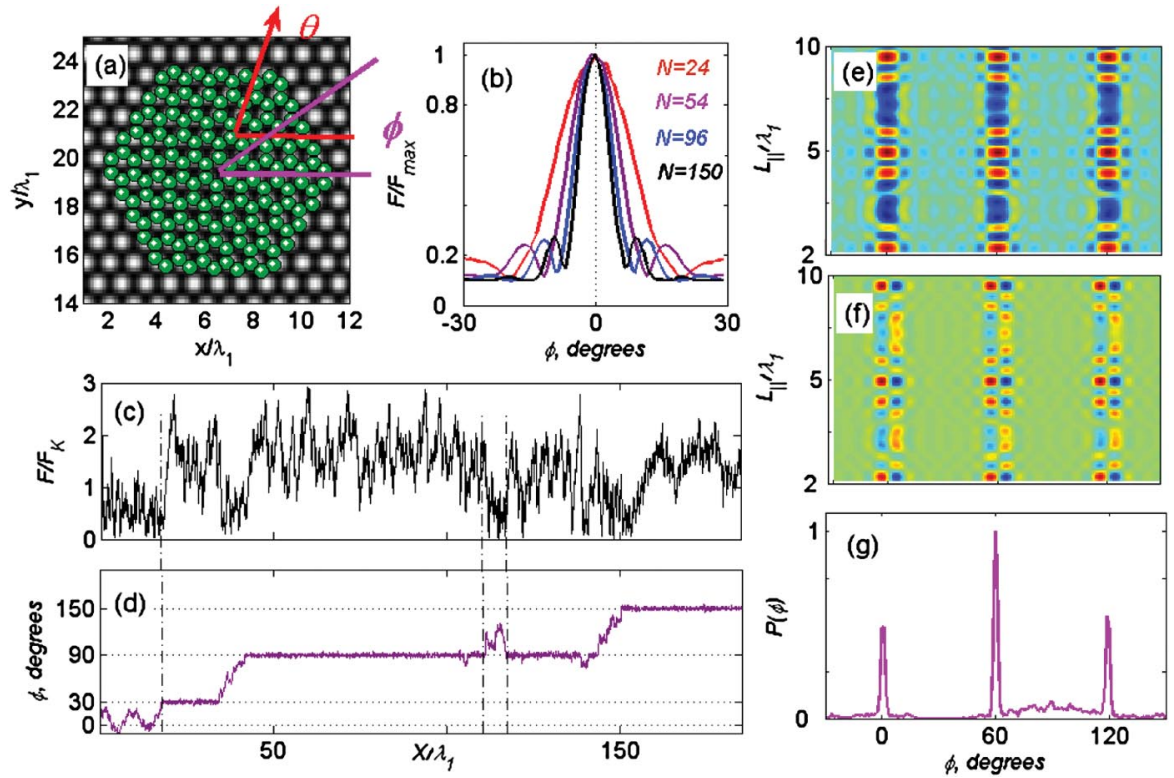

FIG. 2 (color). The 150-atom symmetric graphite flake on the graphite surface. (a) Schematic view of the system. (b) Time-averaged friction force as a function of the misfit angle $\phi$ for nonrotating flakes of different sizes. (c),(d) Frictional force in the direction of pulling and the misfit angle as functions of the support displacement, $X$, for the rotating flake. Dash-dotted lines indicate a few instances where the flake enters or leaves commensurate orientations. (e),(f). 2D maps for the potential and torque experienced by the flake as functions of $\phi$ and the flake displacement in the pulling direction, $L_{\|} . f$. Distribution function for the misfit angle $\phi$ calculated for hundred trajectories, one of which is shown in Figs. 2(c) and 2(d). Parameter values: $F_{K}=K \lambda_{1}, N U_{0} /\left(F_{K} \lambda_{1}\right)=2.5, \gamma V / F_{K}=$ $0.33, \Gamma V /\left(F_{K} \lambda_{1}^{2}\right)=0.13, F_{0} /\left(F_{K} \lambda_{1}\right)=1.25$. Under the experimental conditions, where $K=1.8 \mathrm{~N} / \mathrm{m}, \lambda_{1}=0.246 \mathrm{~nm}, V=$ $30 \mathrm{~nm} / \mathrm{s}$, we have $F_{K}=0.44 \mathrm{nN}, N V_{0}=0.34 \mathrm{eV}, \gamma=4.8 \times 10^{-3} \mathrm{~kg} / \mathrm{s}, \Gamma=1.2 \times 10^{-4} \mathrm{~kg} \mathrm{~nm}^{2} / \mathrm{s}, F_{0}=0.14 \mathrm{nNnm}$. Note that the typical magnitudes of the friction forces obtained in simulations are in agreement with the experimental data presented in Fig. 1. Both experiments and calculations have been performed under overdamped conditions. 
atom in the flake and the graphite surface is approximated by the potential used in Ref. [4],

$$
\begin{aligned}
U(x, y)= & -U_{0}\left[2 \cos \left(2 \pi x / \lambda_{1}\right) \cos \left(2 \pi y / \lambda_{2}\right)\right. \\
& \left.+\cos \left(4 \pi y / \lambda_{2}\right)\right]
\end{aligned}
$$

with $\lambda_{1}=0.246 \mathrm{~nm}$ and $\lambda_{2}=0.426 \mathrm{~nm}$ are periods of the graphite surface, and $U_{0}$ is a constant. Then the rigid flakesurface interaction potential $U_{\text {flake }}\left(x_{c}, y_{c}, \phi\right)$ is simply obtained by summing $U(x, y)$ over $N$ atomic contributions. In order to include the effects of energy barriers which resist the rotation of the flake with respect to the tip, we introduced in Eq. (2), in addition to a "viscous" term, $\Gamma \dot{\phi}$, a frequency-independent, internal friction torque, $-F_{0} \operatorname{sgn}(\dot{\phi})$, analogous to the known static friction. The effect of thermal fluctuations on the translational and rotational motion of the flake is given by a random force and torque, $f_{r_{c}}(t)$ and $f_{\phi}(t)$, which are $\delta$-function correlated, $\left\langle f_{r_{c}, i}(t) f_{r_{c}, j}\left(t^{\prime}\right)\right\rangle=2 \gamma k_{B} T \delta\left(t-t^{\prime}\right) \delta_{i, j}$ and $\left\langle f_{\phi}(t) f_{\phi}\left(t^{\prime}\right)\right\rangle=$ $2 \Gamma k_{B} T \delta\left(t-t^{\prime}\right)$, where $i, j=x, y$.

First, we show in Fig. 2(b) the time-averaged friction force as a function of the misfit angle $\phi$, calculated using Langevin Eq. (1) for rotationally locked symmetric flakes of different sizes, one of which corresponds to Fig. 2(a). The flakes exhibit $60^{\circ}$ rotational symmetry, and therefore the results are presented over a $60^{\circ}$ range of the angles only. In order to compare different flakes the forces are normalized to the maximal value for each flake. The friction force is maximal for a misfit angle of $\phi=0^{\circ}$, for which the lattices of flake and substrate form a commensurate structure, and it is much lower for most other orientations. The angular width of the friction maxima decreases with the size of the flake. These results are in agreement with the experimental data [3-5] and previous quasistatic calculations [4] which provided firm evidence for superlubricity.

However, the flake driven along the surface experiences the influence of the torque and may change its orientation. Including the flake rotation which is governed by Eq. (2), changes qualitatively the dynamics of friction, as shown in Fig. 2(c). Preparing the contact in the superlubric state $\left(\phi=-10^{\circ}\right)$, we found that initially the flake exhibits a superlubric sliding motion with low friction, but after some lag time it performs an erratic stick-slip motion characterized by higher friction. The origin of this phenomenon becomes obvious when one looks at the variation of the misfit angle $\phi$ during the lateral displacement of the flake shown in Fig. 2(d). One can see that after a lag time, which depends on the value of the internal friction $F_{0}$, the flake starts changing its orientation. It should be noted that for $F_{0}>5 K \lambda_{1}^{2}$ the flake remains trapped in the initial state on the time scale of our simulations. In the experiment, such trapping has been the exception, rather than the rule. Nonrotating flakes have been observed only on "highgrade" graphite substrates.
The dynamics of the flake rotation can be represented as a set of alternating segments of rotational stick and slip motion. During the stick intervals the flake is locked in one of the commensurate configurations, with misfit angles $\phi_{\text {com }}=60^{\circ} \times n$, where $n=0, \pm 1, \pm 2, \ldots$ The slip intervals correspond to the escape from a trapped state and the return to either the same or to an adjacent trapped state. The slip events are caused by strong fluctuations of the torque which arise from the stick-slip motion in the lateral direction and from the thermal fluctuations. Thus, the flake performs a correlated stick-slip motion along both translational and rotational coordinates.

Figure 2(e) shows the two-dimensional (2D) map of the potential, which describes the interaction between the flake with a given orientation and the substrate, as a function of the flake displacement along the pulling direction $L_{\|}$and the misfit angle $\phi$. Regions corresponding to high and low values of the potential are displayed by red and blue colors, respectively, with the color scale running from light green for zero potential to full red (blue) for maximum (minimum) values of the potential. One can see that the trapped, commensurate configurations correspond to the cases of the most corrugated potential in the pulling direction. As a result we observe a stick-slip motion for these configurations, as shown in Fig. 2(c). Outside the stripes, which are located around $\phi=\phi_{\text {com }}$, the potential is almost flat, and the flake performs a "smooth" sliding in incommensurate contact with the substrate.

In order to clarify the nature of the rotational motion we present in Fig. 2(f) the 2D map of the torque experienced by the flake with a given orientation in the $\left(L_{\|}, \phi\right)$ space. Here regions of anticlockwise and clockwise torque are displayed by red and blue colors, respectively, and the color scale is the same as in Fig. 2(e). Regions between the stripes, which are located around $\phi=\phi_{\text {com }}$, are characterized by low values of the torque, and in these regions the rotation motion has a diffusive nature, as one can see from Fig. 2(d). However, when the flake configuration is brought close to the commensurate one (falls into the stripes) the torque prevents the escape from the commensurate state and stabilizes the stick-slip motion [see Fig. 2(f)]. Thus, when starting in experiments and in simulations from an incommensurate configuration, the flake approaches one of the stable, commensurate configurations diffusively, and stays there until strong enough fluctuations kick it out of the stripes and bring it back into a diffusion region. Our simulations demonstrate that superlubric states of flakes that are not rotationally locked to the tip are in a sense "free" with respect to the action of the torque while, in contrast, the states with a high friction are the "stable" ones.

The probability distribution function (PDF) for the misfit angles calculated for hundred time series of $\phi$, which are similar to that shown in Fig. 2(d), provides quantitative evidence that the flake spends most time in the locked, commensurate configurations. The PDF was normalized to 

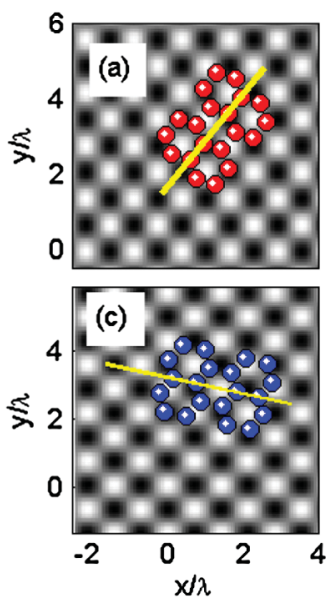
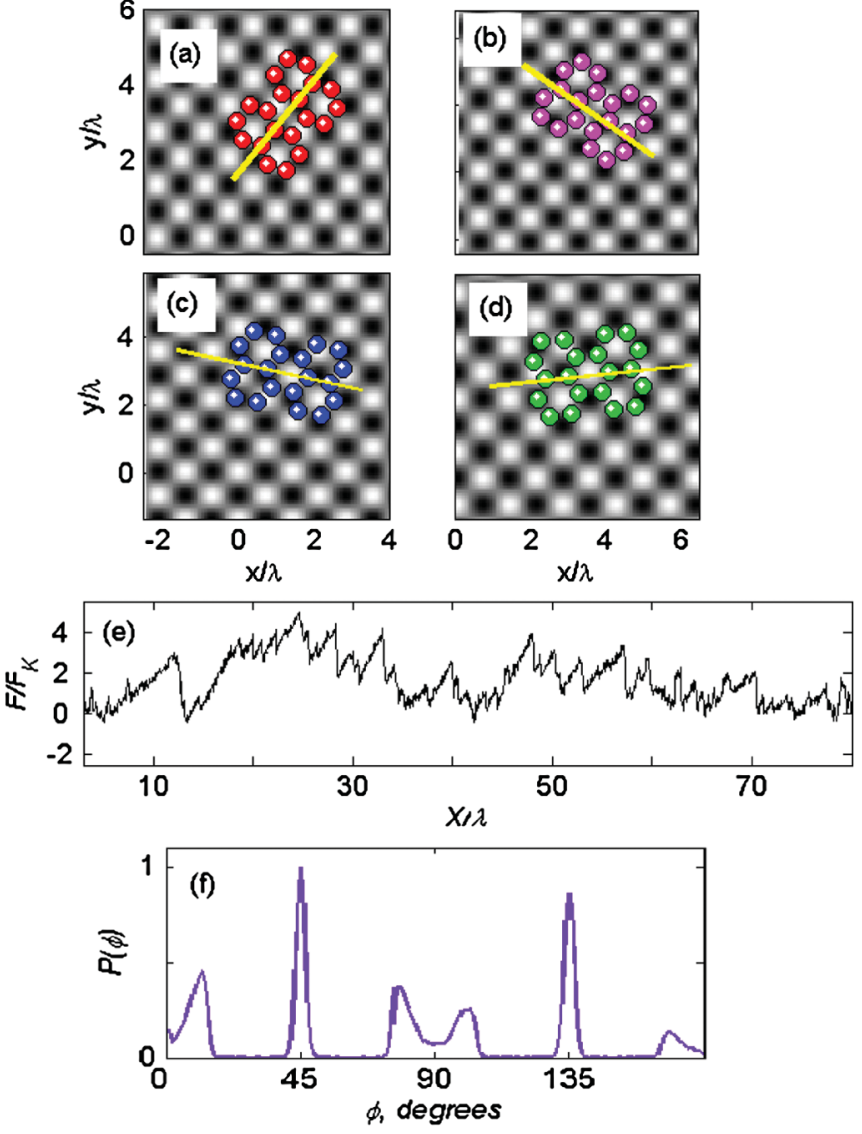

FIG. 3 (color). An asymmetric graphite flake on a surface of square symmetry. (a)-(d) Schematic view of four configurations corresponding to the most commensurate states of the flake. (e) Frictional force in the direction of pulling as a function of the support displacement, $X$, for the rotating flake. (f) Distribution function for the misfit angle $\phi$ calculated for hundred trajectories, one of which is shown in Fig. 3(e). Parameter values as in Fig. 2, and the period of the surface lattice $\lambda=\lambda_{1}$.

its maximal value, and differences in heights and widths of the maxima are the result of the limited statistics.

We found that the above conclusions obtained for symmetric graphite flakes are robust and hold for a broad range of systems. We show in Fig. 3 the results obtained for an asymmetric graphite flake which is driven along a foreign surface with a square symmetry. As in the previous case, we find that starting from a fully incommensurate configuration with low friction the flake, after some lag time, finds itself in a more commensurate state with higher friction. Unlike the symmetric flake, here for the flake pulled at the angle $\theta=70^{\circ}$ we find four different quasicommensurate configurations which are shown in Figs. 3(a)-3(d). It should be noted that for the flake pulled along one of the substrate axes of symmetry the four different configurations merge into two degenerate ones. The PDF for the misfit angle in Fig. 3(f) shows that the flake spends most of its time in the quasicommensurate states performing a correlated stick-slip motion in lateral and rotational dimensions.
Our calculations demonstrate that even in the case of a disordered flake the main conclusions of this Letter hold [27]. Namely, the flake performs reorientations during the translational motion and finds "optimal" configurations which exhibit a stick-slip motion with high friction. Based on our observations, we propose to overcome this enhancement in friction by fixing the flake orientations to ensure persistent incommensurability.

M.D. and J.W.M.F. thank the "Nederlandse Organisatie voor Wetenschappelijk Onderzoek" for financial support. J.K. and M. U. acknowledge the support by the German Research Foundation via Grant Ha No. 1517/ 26-1-3. M. U. acknowledges the support by the Israel Science Foundation (Grant No. 1116/05).

[1] M. Hirano and K. Shinjo, Phys. Rev. B 41, 11837 (1990).

[2] M. Hirano and K. Shinjo, Surf. Sci. 283, 473 (1993).

[3] M. Dienwiebel et al., Phys. Rev. Lett. 92, 126101 (2004).

[4] G.S. Verhoeven, M. Dienwiebel, and J. W. M. Frenken, Phys. Rev. B 70, 165418 (2004).

[5] M. Dienwiebel et al., Surf. Sci. 576, 197 (2005).

[6] M. Hirano et al., Phys. Rev. Lett. 78, 1448 (1997).

[7] P. E. Sheehan and C. M. Lieber, Science 272, 1158 (1996).

[8] Ph. Depondt, A. Ghazali, and J.-C. S. Lévy, Surf. Sci. 419, 29 (1998).

[9] G. He, M.H. Müser, and M. O. Robbins, Science 284, 1650 (1999).

[10] G. He and M.O. Robbins, Phys. Rev. B 64, 035413 (2001).

[11] M. H. Müser, L. Wenning, and M. O. Robbins, Phys. Rev. Lett. 86, 1295 (2001).

[12] C. Daly, J. Zhang, and J. B. Sokoloff, Phys. Rev. Lett. 90, 246101 (2003).

[13] M. H. Müser, Europhys. Lett. 66, 97 (2004).

[14] J. M. Martin et al., Phys. Rev. B 48, 10583 (1993).

[15] J. A. Heimberg et al., Appl. Phys. Lett. 78, 2449 (2001).

[16] M. H. Müser, M. Urbakh, and M. O. Robbins, Adv. Chem. Phys. 126, 187 (2003).

[17] M. Urbakh et al., Nature (London) 430, 525 (2004).

[18] T. Gyalog et al., Europhys. Lett. 31, 269 (1995).

[19] V. Zaloj, M. Urbakh, and J. Klafter, Phys. Rev. Lett. 82, 4823 (1999).

[20] A. Socoliuc et al., Phys. Rev. Lett. 92, 134301 (2004).

[21] E. Gnecco et al., Phys. Rev. Lett. 84, 1172 (2000).

[22] Y. Sang, M. Dube, and M. Grant, Phys. Rev. Lett. 87, 174301 (2001).

[23] O. Dudko et al., Chem. Phys. Lett. 352, 499 (2002).

[24] S. Yu. Krylov et al., Phys. Rev. E 71, 065101 (2005).

[25] M. G. Rozman, M. Urbakh, and J. Klafter, Phys. Rev. E 57, 7340 (1998).

[26] A. Socoliuc et al., Science 313, 207 (2006).

[27] See EPAPS Document No. E-PRLTAO-100-081803 for results obtained for a disorder flake. For more information on EPAPS, see http://www.aip.org/pubservs/epaps.html.

[28] M. Dienwiebel et al., Rev. Sci. Instrum. 76, 043704 (2005). 